\title{
Simulating seismic impacts using vibrating machines
}

\author{
Avetik Abovyan* \\ Moscow State University of Civil Engineering, Yaroslavskoe shosse, 26, Moscow, 129337, Russia
}

\begin{abstract}
The article presents a method to reproduce seismic impacts on structures according to an accelerogram of an earthquake. Three powerful vibrating machines have to be installed on different floors of the structure, where oscillation characteristics are selected based on the spectrum of acceleration of the earthquake and so that the values of the inertial forces arising in the structure during their operation are equal to the true values of seismic forces which arise during the same earthquake. Key words: vibrating machine, eccentric, accelerogram, structure, model.
\end{abstract}

\section{Introduction}

Despite the significant success of domestic and foreign research in the field of seismicresistant construction, the problem of ensuring the safety of buildings and structures during major earthquakes is still far from complete. The complexity of the problem of seismic impact on buildings and structures leads to using a variety of approaches to its solution. The most rational representation of the actual seismic impact can be obtained through experimental studies, where the accuracy of the results largely depends on the method of simulating the seismic load. The methods that are currently used for vibration excitation in the foundation, directional underground detonation or a seismic platform with programmable control are related with major technical and organizational difficulties.

Taking this in to consideration, the development of new and improving existing methods of experimental reproduction about the seismic impact on buildings and structures are appearing as problems to the resolution of which this work is dedicated.

During the oscillations of high-rise buildings, studying their reactions when higher forms of oscillations are overlapping has a certain practical significance from the point of view of improving their calculation for seismic impacts. The articles which are devoted to this question, for example [1,2,3,4,5,6,7], are of theoretical nature.

Here we propose the method of experimental simulation of seismic impacts by using vibrating machines based on the accelerogram of an earthquake.

Vibrating machines are installed on the test structure, which reproduces oscillations of any frequency and stress state in the structure by changing the rotation speed of eccentrics and their masses, including resonance oscillations, in which various types of free oscillations arise in the tested structure.

\footnotetext{
${ }^{*}$ Corresponding author: abovyan_1958@mail.ru
} 
Experimental study of seismic resistance of buildings and structures by using vibrating machines is more accessible, because in this case, complex installations are not required, and this method of simulating the seismic load is acceptable for testing both full-scale structures and their models.

\section{Methods}

During seismic impacts, buildings and structures oscillate in three mutually perpendicular directions and are subjected to a complex stress-strain state. The most dangerous are the two horizontal constituents of the oscillation which in individual directions represent a complex oscillatory motion.

Special experiments were carried out to simulate seismic impacts by creating a complex seismic type dynamic load on the model of a 9-storey steel frame of the building, which is 6 times smaller than the original $[8,9,10,11,12,13,14]$.

For this purpose, a special experimental facility [15] was designed and assembled, which allows for the implementation of complex oscillating motions of seismic type in building models, in the form of overlapping of several modes of free oscillations in one and two mutually perpendicular directions.

The facility consists of a model of a steel framework of a nine-story building, reinforced floor, 6 directional oscillating machines, equipment structures for the engines of the oscillating machines, console, measuring outfit and other fixtures (see figure).

Seismic calculations of high-rise buildings are mainly limited to the first three forms of free oscillations. In this regard, for the practical application of the proposed method of simulating seismic impacts using a system of vibrating machines is limited to the study of the joint operation of three vibrating machines.

Vibrating machines were installed on the $3^{\text {rd }}, 6^{\text {th }}$ and $9^{\text {th }}$ floors of the model. The mounting locations of vibrating machines are aligned with the maximum ordinate, the deflection curve of the relevant mode of oscillation, which made it possible for efficient utilization of power capabilities of vibrating machines.

Each of the three vibrating machines were used to create only one form of resonant oscillation on the test structure.
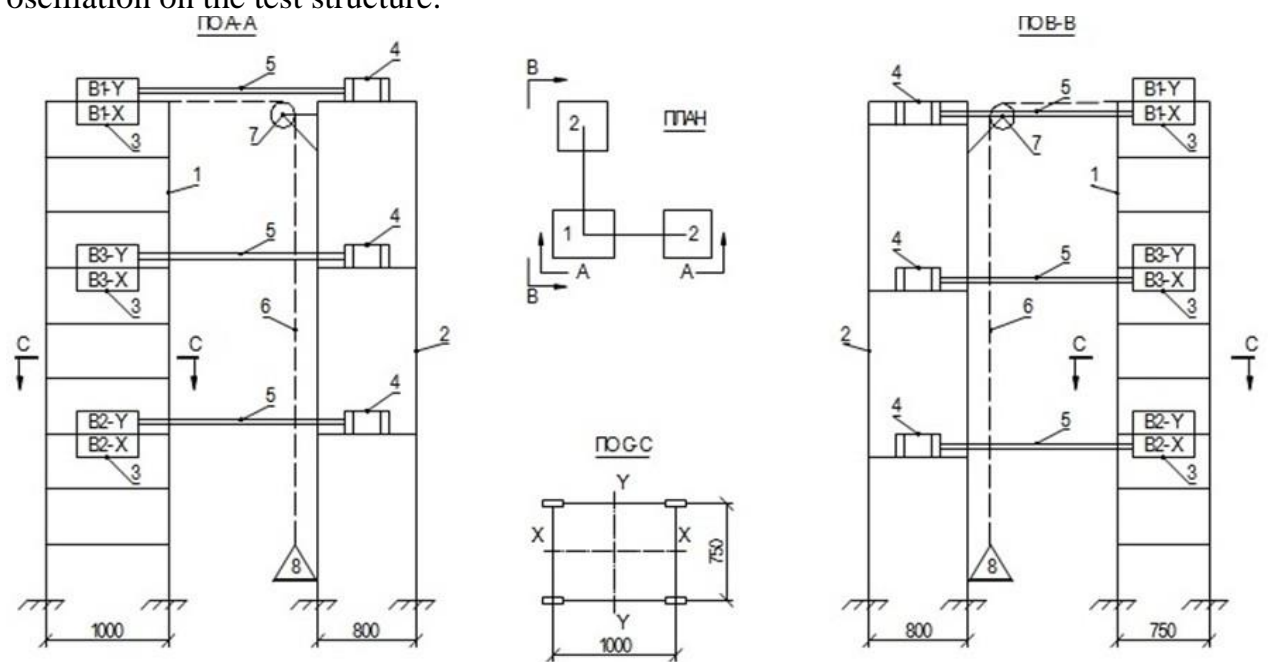

Fig. 1. Construction arrangement of the facility

1 - model, 2 - equipment structures for the engines, 3 - oscillating machines, 4 - engine, 5 - flexible shaft, 6 - cable, 7 - block, 8 - load platform 
This way the vibrating machines B1-Y, B2-Y, B3-Y, which are installed above the floor of the model (see fig.), excited resonant oscillations I, II and III modes respectively along the "Y" axis, and vibrating machines B1-X, B2-X, B3-X, installed below the floor, excited similar oscillations along the " $\mathrm{X}$-axis.

\section{Results}

To simulate the impact of a particular earthquake, it is necessary to select the force parameters of the vibrating machines which corresponds to its accelerogram, which excites the oscillations in the test structure.

The pulsating forces of the vibrating machines installed on the test structure vary according to the harmonic law.

where $m_{e}$ - eccentric mass

$$
F=m_{e} b_{e} \theta_{r}^{2} \sin \theta t=A_{v} \sin \theta t
$$

$b_{e}$ - radius of eccentrics

$\theta_{r}$ - circular frequency

$A_{v}$ - amplitude of the $v^{\text {th }}$ floor

$A_{v}=m_{e} b_{e} \theta_{r}$

When simulating a seismic load, the operating mode of vibrating machines is resonant, therefore, to simulate a particular form of oscillation in a structure, it is necessary to choose the required eccentric mass; $m_{e}$.

To solve this problem, the oscillations of a building or structure were considered according to the accelerogram of earthquakes $y_{0}^{\prime \prime}(t)$ and the values of inertial seismic forces $S_{k}(t)$ were determined. Then the same structure was studied for constrained oscillations from impact of vibrating machines installed on the relevant floor and the inertial forces $S_{k}^{v}(t)$ were determined.

From the condition of equality of the received inertial forces

$$
S_{k}(t)=S_{k}^{v}(t)
$$

The required value of eccentric mass; $m$ of the vibrating machine for a separate given form of its free oscillation of the structure is determined.

The design scheme of the building is considered as a weightless bar with concentrated masses at interfloor levels which move along mutually parallel planes with oscillations of the structure as in the theory of seismic resistance.

The equation of constrained oscillations of the selected system under the impact of an external force $\mathrm{F}(\mathrm{t})$;

$$
\begin{gathered}
m_{1} y_{1}^{\prime \prime}+a_{1} y_{1}+\mu_{1} a_{1} y_{1}^{\prime}-a_{2}\left(y_{2}-y_{1}\right)-\mu_{2} a_{2}\left(y_{2}^{\prime}-y_{1}^{\prime}\right)=-F_{1}(t) \\
m_{2} y_{2}^{\prime \prime}+a_{2}\left(y_{2}-y_{1}\right)+\mu_{2} a_{2}\left(y_{2}^{\prime}-y_{1}^{\prime}\right)-a_{3}\left(y_{3}-y_{2}\right)-\mu_{3} a_{3}\left(y_{3}^{\prime}-y_{2}^{\prime}\right)=-F_{2}(t) ; \\
------- \\
m_{k} y_{k}^{\prime \prime}+a_{k}\left(y_{k}-y_{k-1}\right)+\mu_{k} a_{k}\left(y_{k}^{\prime}-y_{k-1}^{\prime}\right)-a_{k+1}\left(y_{k+1}-y_{k}\right)-\mu_{k+1} a_{k+1}\left(y_{k+1}^{\prime}-y_{k}^{\prime}\right)= \\
=-F_{k}(t) ;
\end{gathered}
$$




$$
\begin{aligned}
& m_{v} y_{v}^{\prime \prime}+a_{v}\left(y_{v}-y_{v-1}\right)+\mu_{v} a_{v}\left(y_{v}^{\prime}-y_{v-1}^{\prime}\right)-a_{v+1}\left(y_{v+1}-y_{v}\right)-\mu_{v+1} a_{v+1}\left(y_{v+1}^{\prime}-y_{v}^{\prime}\right)= \\
& =-F_{v}(t) \text {; }
\end{aligned}
$$

$$
m_{n} y_{n}^{\prime \prime}+a_{n}\left(y_{n}-y_{n-1}\right)-\mu_{n} a_{n}\left(y_{n}^{\prime}-y_{n-1}^{\prime}\right)=-F_{n}(t) ;
$$

Where

$$
\begin{aligned}
& m_{k^{-}} \text {- concentrated mass of the floor } \mathrm{k}, \mathrm{k}=1,2,3, \ldots \ldots, \mathrm{n} \\
& y_{k} \text { - horizontal displacement of the corresponding floor, } \mathrm{y}_{\mathrm{n}+1}=0 \\
& a_{k} \text { - rigidity of the floor } \mathrm{k}, \mathrm{a}_{\mathrm{n}+1}=0 \\
& \mu_{k} \text { - coefficient of viscous resistance of the corresponding floor }
\end{aligned}
$$

On the right hand side of the equation (3) the oscillations of constraining forces of the system $\mathrm{F}(\mathrm{t})$ are replaced by the inertial force of seismic impact $\mathrm{m}_{\mathrm{k}}\left(\mathrm{y}_{\mathrm{k}}{ }^{\text {" }}+\mathrm{y}_{0}{ }{ }^{\prime}\right)$ and when accepting =const, as shown in article [16] for the displacements $y_{k}(t)$ and seismic forces $\mathrm{S}_{\mathrm{k}}(\mathrm{t})$, the following solutions are obtained:

$$
\begin{gathered}
Y_{k}(t)=\sum_{r=1}^{n} \eta_{k r}\left(\frac{T_{r}}{2 \pi}\right)^{2} \tau\left(T_{r}, \delta_{r}, t\right), \\
S_{k}(t)=m_{k}\left(y_{k}^{\prime \prime}+y_{0}^{\prime \prime}\right)=m_{k} \sum_{r=1}^{n} \eta_{k r} \tau\left(T_{r}, \delta_{r}, t\right),
\end{gathered}
$$

where $\quad \tau\left(T_{r}, \delta_{r}, t\right)=\frac{2 \pi}{T_{r}} \int_{0}^{t} y_{0}^{\prime \prime}(\xi) e^{-\frac{\delta_{r}}{T_{r}}(1-\xi)} \sin \frac{2 \pi}{T_{r}}(t-\xi) d \xi$

$\tau$ - acceleration of a linear oscillator within the period $\mathrm{T}_{\mathrm{r}}$ and the decrement of oscillation $\delta_{r}=\mu_{\mathrm{k}}\left(2 \pi^{2} / T_{r}\right)$

$\eta_{k r}$ - coefficient of the shape of the oscillation

$$
\eta_{k r}=C_{k r} \frac{\sum_{i=1}^{n} m_{i} C_{i r}}{\sum_{i=1}^{n} m_{i} C_{i r}^{2}}
$$

where $C_{k r^{-}}$amplitude, which is determined from the following system of free-oscillation equations, therefore

$$
-m_{k} \omega_{r}^{2} C_{k r}+a_{k}\left(C_{k r}-C_{k-1, r}\right)-a_{k+1}\left(C_{k+1, r}-C_{k r}\right)=0
$$

$\omega_{r}$ - the circular frequency of the natural oscillations of the system in the form $r$ 
Maximum values of seismic forces for each form of oscillations that are reached at different times are expressed by the formula:

$$
S_{k r}^{\max }=m_{k} \eta_{k r}\left[\frac{2 \pi}{T_{r}} \int_{0}^{t} y_{0}^{\prime \prime}(\xi) e^{-\frac{\delta_{r}}{T_{r}}(1-\xi)} \sin \frac{2 \pi}{T}(t-\xi) d \xi\right]_{\max }=m_{k} \eta_{k r} \tau_{\max }\left(T_{r}, \delta_{r}\right)
$$

Constrained forces $\mathrm{F}(\mathrm{t})$ are on the right-hand side of equation (3), by replacing the force of the vibrating machines on the $v^{\text {th }}$ floor we obtain that $F_{v}(t)=A_{v} \operatorname{Sin} \theta t$ (assuming $F_{k}(t)=$ 0 on the remaining floors) and by solving them we obtain the following expressions for the displacements $y_{k}^{v}(\mathrm{t})$ and inertial forces $S_{k}^{v}(\mathrm{t})$ :

$$
\begin{gathered}
Y_{k}^{V}(t)=\sum_{r=1}^{n} \eta_{k r}^{V} \frac{\sin (\theta t-\lambda)}{\sqrt{\left(\omega_{r}^{2}-\theta^{2}\right)^{2}+\left(\delta_{r} / \pi\right)^{2} \omega_{r}^{2} \theta^{2}}} \\
\text { where } \eta_{k r}^{V}=C_{k r} \frac{C_{v r} A_{v}}{\sum_{i=1}^{n} m_{i} C_{i r}^{2}} \\
S_{k}^{V}(t)=-m_{k} y_{k}^{\prime \prime}=-m_{k} \sum_{r=1}^{n} \eta_{k r}^{V} \frac{\theta^{2} \sin (\theta t-\lambda)}{\sqrt{\left(\omega_{r}^{2}-\theta^{2}\right)^{2}+\left(\delta_{r} / \pi\right)^{2} \omega_{r}^{2} \theta^{2}}}
\end{gathered}
$$

Considering the fact that when calculating the seismic impact on multi-storey buildings it is essential to take into account the first three forms of oscillations $v_{1}, v_{2}$ and $v_{3}$, we will study the case when the vibration machines are installed on three different floors (see fig.), And simultaneously excite harmonic oscillations of type $A_{v 1} \sin \theta_{1} t, A_{v 2} \sin \theta_{2} t$, $A_{v 3} \sin \theta_{3} t$.

In this case, to determine the inertial (seismic) forces, we have the following equation:

$$
S_{k}^{V}(t)=-m_{k} \sum_{r=1}^{n} C_{k r} \frac{\sum_{j=1}^{3} A_{v j} C_{v j r} \theta_{j}^{2} \sin \left(\theta_{j} t-\lambda_{j}\right)}{\left(\sum_{i=1}^{n} m_{i} C_{i r}^{2}\right) \sqrt{\left(\omega_{r}^{2}-\theta_{i}^{2}\right)^{2}+\left(\delta_{r} / \pi\right)^{2} \omega_{r}^{2} \theta_{i}^{2}}}
$$

In the resonant mode in the form $\mathrm{r}, \omega_{r}=\theta_{j}$ of the oscillation, the influence of the members of the series (12) could be neglected, and then for the maximum values $\omega_{r} \neq \theta_{j}$ of the inertial (seismic) forces for form $r$ oscillations, we obtain:

$$
S_{k r}^{V(\max )}=\frac{m_{k}}{\delta_{r} / \pi} \frac{A_{v} C_{v r} C_{k r}}{\sum_{i=1}^{n} m_{i} C_{i r}^{2}}
$$

According to the accepted condition (2), in order to reproduce an earthquake in form $r$ of oscillation by using vibrating machines installed on $v^{\text {th }}$ floor, it is necessary that the following equality takes place. According to the expressions (9) and (14) 


$$
m_{k} \eta_{k r} \tau_{\max }\left(T_{r}, \delta_{r}\right)=\frac{m_{k}}{\delta_{r} / \pi} \frac{A_{v} C_{v r} C_{k r}}{\sum_{i=1}^{n} m_{i} C_{i r}^{2}}
$$

where substituting from equation (1) during resonance

$$
A_{v}=m_{e} b_{e} \theta^{2}=m_{e} b_{e}\left(\frac{2 \pi}{T_{r}}\right)^{2}
$$

and $\tau\left(T_{r}, \delta_{r}\right)$ the values of formula (6).

We determine the required eccentric mass of the vibrating machine depending on the earthquake accelerogram $y_{0}^{\prime \prime}(\mathrm{t})$ and on the dynamic characteristics and the tested structure $\mathrm{T}_{\mathrm{r}}$ and $\delta_{\mathrm{r}}$

$$
m_{e}=\frac{\frac{\delta_{r}}{\pi} \sum_{i=1}^{n} m_{i} \frac{C_{i r}}{C_{v r}}\left[\frac{2 \pi}{T_{r}} \int_{0}^{t} y_{0}^{\prime \prime}(\xi) e^{-\frac{\delta_{r}}{T_{r}}(1-\xi)} \sin \frac{2 \pi}{T_{r}}(t-\xi) d \xi\right]_{\max }}{\left(\frac{2 \pi}{T_{r}}\right)^{2} b_{e}}
$$

The resulting expression $m_{e}$ is substituted in $A_{v j}$ in formula (13) and considering the resonant mode of operation of vibrating machines $\left(\omega_{r}=\theta\right)$, neglecting the phase shift of their work, we obtain the following expression to determine the inertial (seismic) forces arising in the structure from the impact of vibration machines.

$$
S_{k}^{V}(t)=-m_{k} \sum_{r=1}^{n} \eta_{k r} \tau_{\max }\left(T_{r} \delta_{r}\right) \sin \frac{2 \pi}{T_{r}} t
$$

\section{Discussion}

Experimental studies of seismic resistance of buildings and structures on the proposed method of simulating seismic effects should be carried out as follows:

- Conduct a calculation-theoretical analysis of the reaction (stress-strain state) of the test structure according to the chosen accelerogram of the earthquake $y$ "(t);

- Based on the analysis of the reaction of the test structure, determine the number of oscillation modes that need to be taken into account and accordingly, the number of vibrating machines to be taken into account and the corresponding locations for their installation;

- Select the parameters of vibrating machines that excite different forms of oscillations, i.e. their respective power and eccentric masses.

- The value of eccentric masses must be determined by the formula (17);

\section{Conclusions}

Thus, a method has been developed for experimentally reproducing seismic impacts on buildings and structures by simulating their stress-strain state, which occurs during 
earthquakes, using several simultaneously operating vibrating machines installed on the building and excite resonant vibrations on individual forms of free oscillations.

We have solved the problem of determining the eccentric masses of vibrating machines by known dynamic characteristics of the building $(\mathrm{T})$ and the accelerogram of the reproduced earthquake $y$ " $(\mathrm{t})$ under the condition that the maximum values of seismic forces in this form of oscillation according to the accelerogram of the earthquake and vibration excitation coincide.

The developed method of experimental reproduction of seismic impacts allows to investigate the behavior of buildings and structures in various earthquakes for a relatively small material and labor cost with sufficient accuracy for practical purposes.

I express my gratitude to E.E. Khachiyan, academic of NAN RA, doctor of phys. Math. Sciences, prof. director of the department "constructional mechanics" YSUAC

\section{Reference}

1. Av.G. Abovyan, Ar.G. Abovyan, K.K. Petrosyan On the possibility of modeling the seismic load with the simultaneous operation of a system of vibration machines // Bulletin of the Engineering Academy of Armenia. Collection of scientific and technical articles. T. 9, No. 4, Yerevan, (2012). P. 787-791.

2. Av.G. Abovyan, Ar.G. Abovyan, K.K. Petrosyan. Special features when reproducing seismic impacts on building models. // Collection of scientific works of YSUAC "Architecture, town-planning, construction, geodesy. T. 3 (33), Yerevan, (2008). P. 91 93.

3. Av.G. Abovyan, Ar.G. Abovyan, Study of graphs of oscillations of buildings and structures under the impact of real earthquakes. // Collection of scientific papers. T. 2, Yerevan, (2003). C.1-3.

4. Av.G. Abovyan, Ar.G. Abovyan, Dependence of the damping ratio on the oscillation forms of plates. // Collection of scientific papers. T. 3, Yerevan, (2001). P. 5-7.

5. Arakelyan S.G. Some results of the survey of the consequences of the Chernigov earthquake in 1979 // Interuniversity thematic collection of scientific works "Building Constructions", Yerevan, (1987). P.24-27.

6. A.T. Goroyan, E.A. Goroyan To compiling a choice of accelerograms of strong earthquakes for the calculation of structures for seismic impact, taking into account the categories of soils. // Interuniversity thematic collection of scientific works "Investigations on earthquake resistance of structures", Yerevan, (1984). pg 23-28.

7. Av.G. Abovyan The possibility of simulating spatial oscillations of buildings in earthquakes. / / Ed. Arm NIISA Abstracts of the Transcaucasian Conf. Young scientists and specialists in construction and architecture, Yerevan, (1984) $1 \mathrm{~S}$.

8. Av.G. Abovyan, G. A. Abovyan The possibility of creating a complex dynamic load of a seismic type with the simultaneous operational system of vibrating machines. // Journal of the Bulletin of Construction Machinery, No. 2 (1003), Moscow, (2018) Pp. 36-37.

9. Av.G. Abovyan, Ar.G. Abovyan, Petrosyan K.K. Investigation of the stress-strain state of structures with flat and spatial oscillations. // Bulletin of the Engineering Academy of Armenia. Collection of scientific and technical articles. T. 9, No. 3, Yerevan, (2012) pp. 552-554.

10. Av.G. Abovyan, Ar.G. Abovyan, K.K. Petrosyan Method of experimental research of seismic resistance of buildings and structures. // Bulletin of builders of Armenia. №1011 (146-147), Yerevan, (2009) With. 5-6. 
11. Ar.G. Abovyan, the method of simulating the seismic impact on buildings and structures using a system of vibratiing machines. // Collection of scientific articles of YSUAS. T. 2 (24), Yerevan, (2004) P. 76-77.

12. G. A. Abovyan, E.E Khachiyan., S.G. Arakelyan, Av.G. Abovyan Determining the accuracy of simulation of seismic impacts by using vibrating machines. // Interdepartmental collection of scientific and technical articles by faculty, researchers, $\mathrm{PhD}$ "Construction" students, Yerevan, (1997) pp. 27-29.

13. Av.G. Abovyan, Ar.G. Abovyan., The recipe for reliability. // Journal of "Zvartnots" (Industry, construction and architecture of Armenia), No. 8-9, Yerevan, (1991) C. 2.

14. Av.G. Abovyan The method of testing a model of a building structure. Author's certificate No. 4266315 / 31-33 (11762). Decision of the State Technical University of the Russian Federation on (04.02.1988)

15. G. A. Abovyan, Av.G. Abovyan, S.G. Arakelyan Setup and test method for building models for seismic impact. / / Special. Release. Bulletin of builders of Armenia, No. 8 (13), (1997) C.7-8.

16. E.E. Khachiyan Seismic impact on high-rise buildings and structures. / / Ed. "Hayastan", Yerevan, (1973), 327 C. 